\title{
Delivery and neonatal outcomes in women with antepartum severe maternal morbidity: a population-based study.
}

\author{
Mégane Raineau ${ }^{1}$, Catherine Deneux-Tharaux ${ }^{1}$, Aurélien $\mathrm{Seco}^{2}$, and Marie-Pierre Bonnet ${ }^{1}$ \\ ${ }^{1}$ INSERM \\ ${ }^{2}$ INSERM U1153
}

May 12, 2021

\begin{abstract}
Objectives: To assess the incidence, causes, risk factors and adverse outcomes of antepartum severe maternal morbidity (SMM) Design: Population-based case-control study Setting: 119 Maternity hospitals, 6 French regions Population: All women with antepartum SMM (cases, $\mathrm{N}=601$ ), a randomly selected control sample of women who gave birth without SMM in the same hospitals during the same period (controls, $\mathrm{N}=3650$ ) Methods: Uni- and multivariable logistic regression with multiple imputation Main outcomes measure: Antepartum SMM, defined as a morbid event occurring from 22 weeks of gestation and before the onset of labour Results: Antepartum SMM complicated $0.33 \%$ of pregnancies (95\%CI, 0.30-0.36). Rates of prematurity, neonatal mortality, and transfer to the neonatal intensive care unit were 10 times higher for babies whose mothers had antepartum SMM than for the control mothers. Similarly, emergency caesarean and general anaesthesia were more frequent in women with antepartum SMM. Risk factors for antepartum SMM were maternal age >35 (aOR 1.55; 95\% CI, 1.22-1.97), increased body mass index (aOR for $5 \mathrm{~kg} / \mathrm{m} 2$ increase, 1.24; 95\% CI, 1.14-1.36), maternal birth in sub-Saharan Africa (aOR, 1.80; 95\% CI, 1.29-2.53), pre-existing medical condition (aOR, 2.56; 95\% CI, 1.99-3.30), nulliparity (aOR, 2.26; 95\% CI, 1.83-2.80), previous pregnancy-related hypertensive disorders (aOR, 4.94; 95\% CI, 3.36-7.26), multiple pregnancy (aOR, 5.79; 95\% CI, 3.75-7.26), irregular prenatal care (aOR, 1.86; 95\% CI, 1.27-2.72). Conclusion: Antepartum SMM is rare but associated with a massively higher incidence of adverse delivery and neonatal outcomes.
\end{abstract}

\section{Hosted file}

Main Document.pdf available at https://authorea.com/users/413418/articles/521740-deliveryand-neonatal-outcomes-in-women-with-antepartum-severe-maternal-morbidity-a-populationbased-study 